\title{
Leptin dysfunction and the risk of dyslipidemia and metabolic syndrome in Romanian HIV- infected patients undergoing antiretroviral therapy
}

\author{
Cătălin Tiliş̧can ${ }^{1,2^{*}}$, Raluca Mihăilescu², Daniela Munteanu' ${ }^{2}$, Victoria Aramă ${ }^{1,2}$, Ana Maria Tudor ${ }^{1,2}$, \\ Cristina Popescu ${ }^{1,2}$, Adriana Hristea ${ }^{1,2}$, Anca Ruxandra Negru², Roxana Petre², Iulia Niculescu ${ }^{1,2}$, Mihai Lazăr ${ }^{1,2}$, \\ Daniela Adriana Ion ${ }^{1}$, Adrian Streinu-Cercel ${ }^{1,2}$, Sorin Ștefan Aramă ${ }^{1}$
}

From The 7th Romanian National HIV/AIDS Congress and The 2nd Central European HIV Forum Sibiu, Romania. 29-31 May 2014

Leptin is a hormone secreted by the adipose tissue that may be associated in the general population with components of the metabolic syndrome (MS). Our objective was to test the association between dyslipidemia, MS presence and circulating leptin dysfunction in a cohort of HIV-infected non-diabetic patients undergoing combinant antiretroviral therapy (cART).

We included HIV-infected non-diabetic consecutive patients undergoing cART, admitted to the National Institute for Infectious Diseases "Prof. Dr. Matei Balş", between 2008-2011. The diagnosis of MS was made using the International Diabetes and American Heart Association harmonized criteria from 2009. Circulating levels of leptin (BioSource EASIA) were measured.

We enrolled 95 patients: 53 (55.8\%) males (mean age $=33.1 \pm 13.4$ years) and $42(44.2 \%)$ females (mean age $=30.5 \pm 13.6$ years). Most patients $(72.5 \%)$ had undetectable HIV viral load; median CD4 count was 493.5 $(\mathrm{IQR}=422) / \mathrm{cmm}$. The median time from HIV diagnosis was 60 (IQR=73) months. The median time on cART was 58.5 (IQR=70) months, $53.8 \%$ of patients had experienced more than one cART regimen.

The prevalence of MS was $17.1 \%$. Elevated blood pressure, elevated waist circumference and abnormal fasting glucose prevalences were $30.3 \%, 17.1 \%$ and $6.5 \%$, respectively.

${ }^{1}$ National Institute for Infectious Diseases "Prof. Dr. Matei Balș", Bucharest, Romania

Full list of author information is available at the end of the article
Median serum leptin was $1.89(\mathrm{IQR}=3.57) \mathrm{ng} / \mathrm{mL}$. Circulating leptin dysfunction was present in almost half of patients, hypoleptinemia being more frequent (42.\%) than hyperleptinemia (8.5\%). Hypoleptinemia was more frequent in men $(62.3 \%)$ comparative to women (17.1\%), $\mathrm{p}=0.000$.

The prevalence of MS in patients with hypoleptinemia was $25.8 \%$ vs $10.8 \%$ in persons with normal leptin values $(\mathrm{p}=0.261)$. Hypoleptinemia was associated with elevated waist circumference $(\mathrm{p}=0.004)$ and abnormal fasting glucose $(\mathrm{p}=0.05)$ in women. More than half $(65.6 \%)$ of men with hypoleptinemia had reduced HDL-cholesterol levels vs $29.4 \%$ in men with normal levels of leptin. As expected, hyperleptinemia was associated with the increase of body mass index, both in men $(p=0.000)$ and women $(\mathrm{p}=0.05)$.

In our cohort of young cART multiexperienced HIV patients leptin dysfunction was not significantly associated with MS presence. Leptin was correlated with several MS components (HDL-dyslipidemia, elevated circumference, abnormal fasting glucose) with significant gender differences, that suggests that leptin may play different roles in the regulation of glucose and lipid metabolism according to the sex.

\section{Authors' details}

'National Institute for Infectious Diseases "Prof. Dr. Matei Balş", Bucharest, Romania. ${ }^{2}$ Carol Davila University of Medicine and Pharmacy, Bucharest, Romania. 


\section{doi:10.1186/1471-2334-14-S4-012}

Cite this article as: Tiliş̧an et al:: Leptin dysfunction and the risk of dyslipidemia and metabolic syndrome in Romanian HIV-infected patients undergoing antiretroviral therapy. BMC Infectious Diseases 2014 14(Suppl 4):012.

Submit your next manuscript to BioMed Central and take full advantage of:

- Convenient online submission

- Thorough peer review

- No space constraints or color figure charges

- Immediate publication on acceptance

- Inclusion in PubMed, CAS, Scopus and Google Scholar

- Research which is freely available for redistribution

Submit your manuscript at www.biomedcentral.com/submit
C Biomed Central 